\title{
Hyperosmolar Hyperglycaemic State and Diabetic Ketoacidosis in Nivolumab-Induced Insulin-Dependent Diabetes Mellitus
}

\author{
Ahmed Osman Saleh ${ }^{1}, \underline{\text { Ruba Taha }}{ }^{2}$, Shehab Fareed A. Mohamed ${ }^{2}$, Mohammed Bashir ${ }^{3}$ \\ ${ }^{1}$ Clinical Fellow, Endocrinology and Diabetes, HMC, Qatar \\ ${ }^{2}$ Hamad Medical Corporation, National Center for Cancer Care and Research, Qatar \\ ${ }^{3}$ Department of Endocrinology, Qatar Metabolic Institute Hamad Medical Corporation, Qatar
}

\section{Doi: 10.12890/2021_002756 - European Journal of Case Reports in Internal Medicine - ๑ EFIM 2021}

Received: 01/07/2021

Accepted: $12 / 07 / 2021$

Published: $13 / 08 / 2021$

How to cite this article: Saleh AO, Taha R, Mohamed SFA, Bashir M. Hyperosmola hyperglycaemic state and diabetic ketoacidosis in nivolumab-nduged insulin-dependent diabetes mellitus. EJCRIM 2021;8: doi:10.12890/2021_002756.

Conflicts of Interests: The authors declare there are no competing interests.

This article is licensed under a Commons Attribution Non-Commercial 4.0 License

\section{ABSTRACT}

Nivolumab is a monoclonal antibody directed against programmed cell death-1 receptor. It has an increasing application in the treatment of various advanced metastatic cancers. The incidence of autoimmune side effects associated with such agents is expected to increase. New-onset autoimmune diabetes mellitus associated with immune checkpoint inhibitor treatment is rare, occurring in less than $1 \%$ of patients. Nivolumab-induced diabetes often presents as diabetic ketoacidosis, which could be life-threatening if not recognized and treated promptly. We present the case of a patient who developed severe diabetic ketoacidosis concomitant with hyperosmolar hyperglycaemic state (HHS) after receiving nivolumab for metastatic testicular lymphoma. Pre-nivolumab blood glucose levels were normal, apart from transient hyperglycaemia related to steroids as part of the chemotherapy protocol. The diagnosis was confirmed with extremely low C-peptide in the clinic.

\section{LEARNING POINTS}

- Checkpoint inhibitor-associated diabetes can present abruptly with life-threatening complications.

- Most patients require multiple daily injections of insulin upon discharge.

- Cessation of checkpoint inhibitor therapy does not revert diabetes.

\section{KEYWORDS}

DKA, HHS, nivolumab, diabetes mellitus, checkpoint inhibitors

\section{CASE DESCRIPTION}

We present a 67-year-old male who presented with a large destructive pelvic mass, testicular mass and severe bone pain limiting his mobility. Subsequently, he was diagnosed with testicular lymphoma stage IV and underwent right orchiectomy. The histopathology showed large B-cell lymphoma, ABC subtype Ki67 80\% with a triple expression of MYC, BCL2 and BCL6 (oncogenes involved in B-cell lymphomas, associated with an aggressive clinical course). No MYC rearrangement. Staging PET-CT showed extensive disease with adrenal, bone and lymph node metastases. The patient was normoglycaemic at that stage. He was initially treated with 6 cycles of R-CHOP (rituximab, cyclophosphamide, hydroxydaunorubicin, Oncovin and prednisone) with 4 intrathecal methotrexate (IT-MTX), 2 extra rituximab and 2 cycles of high-dose MTX. There was no detectable cancer uptake in the follow-up PET scan suggestive of complete metabolic response (CMR). He relapsed 6 months after completing therapy; thus, he received 2 cycles of R-ESHAP (rituximab, etoposide, steroid, high-dose Ara-C and platinum- 
based cisplatin) with a partial response on the PET scan. He was shifted to ICE chemotherapy (ifosfamide, carboplatin and etoposide) to enhance the response and underwent stem cell harvest. Pre-transplant PET-CT showed disease progression; hence he was shifted to 3rdline therapy with nivolumab. PET-CT showed CMR after 4 doses. He had transient steroid-related hyperglycaemia during the chemotherapy cycles (Fig. 1). He was not on antidiabetes medications, and his latest HbA1c before nivolumab was 5.8\%. Ten days following the 7th dose of nivolumab, the patient was admitted with a severe episode of diabetic ketoacidosis (DKA) and hyperosmolar hyperglycaemic state (HHS). He presented with vomiting, abdominal pain and confusion. He was found to have random plasma glucose of $56 \mathrm{mmol} / \mathrm{l}$, bicarbonate of 5.6 $\mathrm{mmol} / \mathrm{l}, \beta$-hydroxybutyrate was $9.6 \mathrm{mmol} / \mathrm{l}, \mathrm{pH}$ of 7.132 and calculated serum osmolality was $336 \mathrm{mOsm} / \mathrm{kg}$. He also had acute kidney injury (creatinine increased from $80 \mu \mathrm{mol} / \mathrm{I}$ to $211 \mu \mathrm{mol} / \mathrm{l}$, urea from $4 \mathrm{mmol} / \mathrm{l}$ to $16 \mathrm{mmol} / \mathrm{l}$ ) and lactic acid of $5.9 \mathrm{mmol} / \mathrm{I}$. He had normal amylase and lipase. He was treated with IV fluid hydration, electrolyte replacement and insulin infusion as per the hospital's local DKA protocol. He was discharged on multiple daily injections of insulin. During outpatient follow-up HbA1c increased from $5.7 \%$ to $8 \%$, C-peptide $<0.02$ ng/ $\mathrm{ml}$, fasting plasma glucose of $12 \mathrm{mmol} / \mathrm{l}$ and insulin level of $0.8 \mathrm{mcunit} / \mathrm{ml}$ (Table 1). One month after the diagnosis of diabetes mellitus, the patient remained on multi-daily injections of insulin, and nivolumab was resumed. His blood sugar is currently controlled on multiple daily insulin injections, waiting for a possible autologous stem cell transplant.

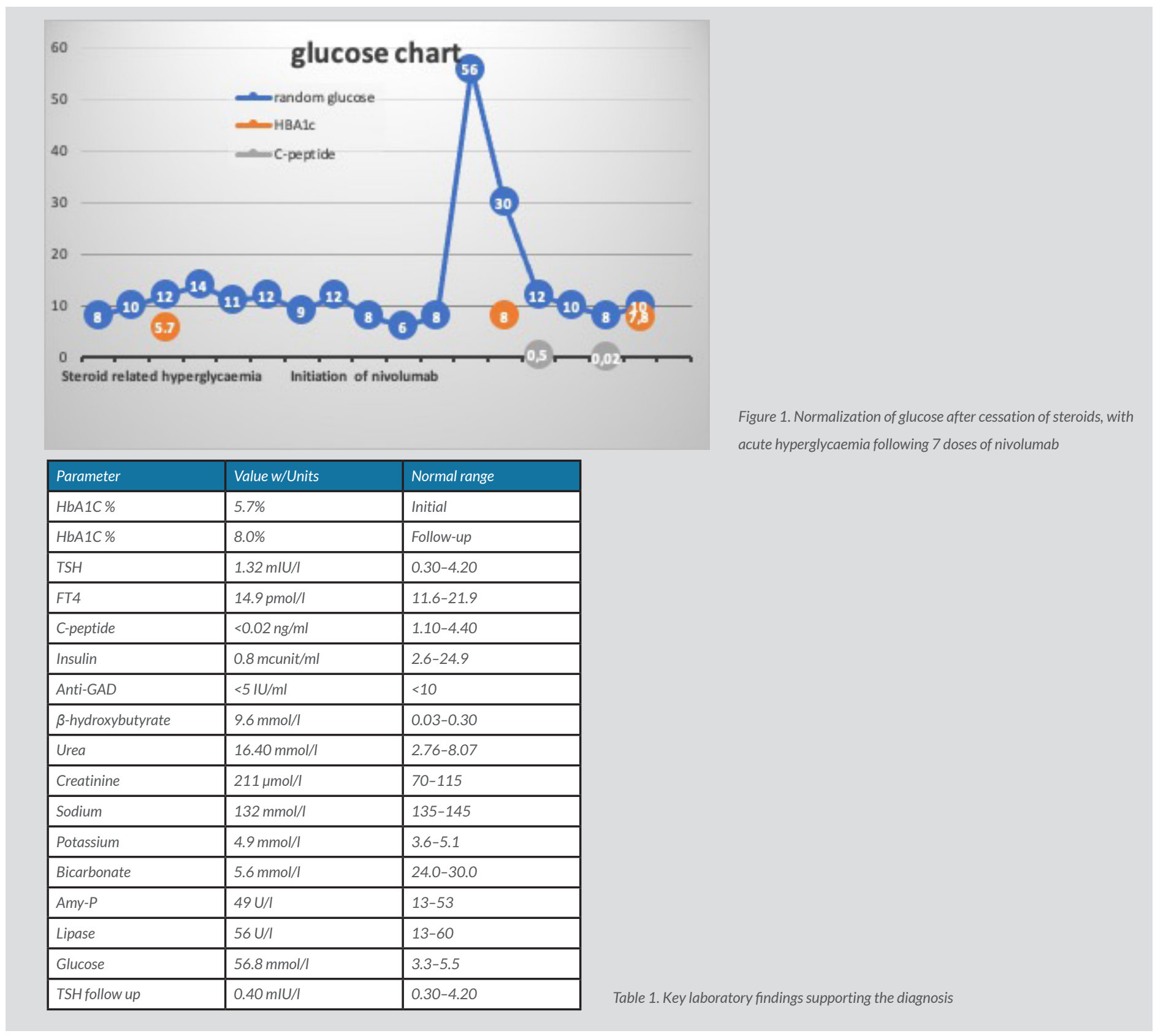




\section{DISCUSSION}

Here, we present a case of rapid-onset diabetes mellitus secondary to insulin deficiency, possibly related to nivolumab therapy in a patient with metastatic testicular lymphoma. After the 7th dose (3 months), the patient presented with severe DKA, HHS, acute kidney injury and a drop in consciousness levels. Insulin deficiency was confirmed in the clinic by low C-peptide levels and low insulin levels, and the absence of anti-GAD antibodies ruled out autoimmune diabetes. The patient was treated with multiple daily insulin injections.

Endocrinopathies are well-recognized immune-related side effects of checkpoint inhibitors; commonly manifesting as thyroiditis, hypophysitis, adrenalitis and, occasionally, diabetes mellitus ${ }^{[1]}$. A systematic review and meta-analysis that included 7,551 patients showed that diabetes mellitus was reported in $0.2 \%$ of patients treated with checkpoint inhibitors ${ }^{[1]}$. Most of the patients with checkpoint inhibitorinduced diabetes presented with DKA as observed in isolated case reports and case series of checkpoint inhibitor-induced diabetes mellitus $[2,3]$. Combination therapy of programmed cell death protein 1 (PD-1) and its ligand, programmed death-ligand 1 (PD-L1), was associated with an overall increased risk of adverse events, including hypothyroidism and diabetes ${ }^{[1]}$. Most patients presented with severe DKA with no glycaemic abnormality before presenting with DKA. Patients with certain HLA genotypes, mostly DRB1*03 or 04, were more susceptible to immune checkpoint inhibitor-induced diabetes; on the other hand, type 2 diabetes mellitus was not associated with an increased risk of autoimmune diabetes ${ }^{[4,5]}$.

Even though definitive associations cannot be ascertained, the characteristics of the presentation of the cases are highly suggestive of a causal association. The improvement in the patient's transient steroid-related hyperglycaemia and normal HbA1c excludes steroid-induced diabetes. The acute presentation with DKA and HHS 3 months after nivolumab therapy supports the diagnosis. A recent systematic review and meta-analysis reported that $76 \%$ of patients who developed new-onset diabetes typically presented with fulminant DKA and rapid beta-cell destruction within an average of 49 days ${ }^{[6]}$. In classical type 1 diabetes, antibodies (anti-GAD antibodies, anti-insulin antibodies, anti-islet cell antibodies) are present in $80-95 \%$ of the cases ${ }^{[7]}$. However, in immunotherapy-related diabetes, anti-GAD antibodies are present in almost half of the cases. In contrast, islet cell antibodies are present in $13 \%$; furthermore, those with antibodies had a more rapid onset than individuals without antibodies [6]. In this case, anti-GAD antibodies were negative. Our patient's HLA genotype was positive for DRB1, but this can only partially explain some individuals with a higher risk of diabetes; thus, it is not practical to recommend HLA genotype screening before immune checkpoint inhibitor therapy. The current knowledge of biomarkers for the stratification of patients remains insufficient. The only way of recognizing patients with diabetes is through blood glucose monitoring and education about symptoms and signs of diabetes mellitus ${ }^{[8]}$.

PD-1 and its 2 ligands, PD-L1 and PD-L2, are widely expressed in immune and non-immune cells and exert a broader range of immune suppression roles in the activation of $\mathrm{T}$ lymphocytes. Their interaction protects tissues from autoimmune attacks and regulates the induction and maintenance of peripheral tolerance ${ }^{[9]}$. Certain cancers such as melanoma and breast cancer express a high level of PD-L1 and use the interaction between PD-1 and PD-L1 to avoid being attacked by the immune system. Checkpoint inhibitors like nivolumab and pembrolizumab have established effectiveness against a variety of advanced cancers but can also trigger immune-related adverse reactions ${ }^{[10,11]}$. Blockade of PD-1 and PD-L1 has been shown to precipitate autoimmune diabetes mellitus in non-obese diabetic mice ${ }^{[12]}$. Anti-PD-1 could have the same effect in humans, and the blocked PD-1 might activate autoreactive T cells, resulting in an autoimmune response against pancreatic islet cells.

Furthermore, studies have demonstrated that patients with type 1 diabetes mellitus had reduced PD-1 expression in CD4+ T cells compared with healthy controls, which may indicate that lower PD-1 expression in CD4+ T cells could contribute to the development of type 1 diabetes mellitus T-cell activation ${ }^{[13]}$. Immune-checkpoint inhibitor treatment results in significant irreversible beta-cell destruction after medication withdrawal, necessitating long-term use of insulin therapy. Only 1 case was reported with reversal of insulin dependence with C-peptide within the normal range throughout the patient follow-up ${ }^{[14]}$. The compelling efficacy of immunotherapy in advanced malignancies and the limited alternative treatment options make stopping immune checkpoint inhibitors in such cases impractical.

\section{CONCLUSION AND RECOMMENDATIONS}

Immune checkpoint inhibitor-induced diabetes mellitus is rare, but its prevalence is expected to rise with the increased use of these agents. Symptoms and signs of hyperglycaemia should be checked during all follow-up visits. While the acute measurement of islet cell autoantibodies is unnecessary, a detailed work-up that includes antibodies and C-peptide is required to establish the correct diagnosis and tailor further treatment as most patients develop irreversible insulin deficiency. Checkpoint inhibitor-induced diabetes mellitus can be a life-threatening adverse event presenting abruptly with DKA. We recommend that all the patients receiving checkpoint inhibitors be educated on the symptoms and signs of hyperglycaemia and should be directed to seek early medical advice if any of these develop. Healthcare providers should be educated about the diagnosis and management of DKA/HHS related to immune checkpoint inhibitors. We recommend a periodic measurement of blood glucose at least before every dose. 


\section{REFERENCES}

1. Barroso-Sousa R, Barry WT, Garrido-Castro AC, Hodi FS, Min L, Krop IE, et al. Incidence of endocrine dysfunction following the use of different immune checkpoint inhibitor regimens. JAMA Oncology 2018;4(2):173.

2. Venetsanaki V, Boutis A, Chrisoulidou A, Papakotoulas P. Diabetes mellitus secondary to treatment with immune checkpoint inhibitors. Curr Oncol 2019;26(1):e111-e114.

3. Wright JJ, Salem J, Johnson DB, Lebrun-Vignes B, Stamatouli A, Thomas JW, et al. Increased reporting of immune checkpoint inhibitor-associated diabetes. Diabetes Care 2018;41(12):e150-e151.

4. Magis Q, Gaudy-Marqueste C, Basire A, Loundou A, Malissen N, Troin L, et al. Diabetes and blood glucose disorders under anti-PD1. J Immunother 2018;41(5):232-240.

5. Akturk HK, Kahramangil D, Sarwal A, Hoffecker L, Murad MH, Michels AW. Immune checkpoint inhibitor-induced Type 1 diabetes: a systematic review and meta-analysis. Diabet Med 2019;36(9):1075-1081.

6. de Filette JMK, Pen JJ, Decoster L, Vissers T, Bravenboer B, Van der Auwera BJ, et al. Immune checkpoint inhibitors and type 1 diabetes mellitus: a case report and systematic review. Eur J Endocrinol 2019;181(3):363-374.

7. Towns R, Pietropaolo M. GAD65 autoantibodies and its role as biomarker of Type 1 diabetes and Latent Autoimmune Diabetes in Adults (LADA). Drugs Future 2011;36(11):847.

8. Higham CE, Olsson-Brown A, Carroll P, Cooksley T, Larkin J, Lorigan P, et al. Society for Endocrinology Endocrine Emergency Guidance: acute management of the endocrine complications of checkpoint inhibitor therapy. Endocr Connect 2018;7(7):G1 -G7.

9. Pu N, Lou W, Yu J. PD-1 immunotherapy in pancreatic cancer. J Pancreatol 2019;2(1):6-10.

10. Spiers L, Coupe N, Payne M. Toxicities associated with checkpoint inhibitors-an overview. Rheumatology 2019;58 Suppl 7:vii7-vii16.

11. Stamatouli AM, Quandt Z, Perdigoto AL, Clark PL, Kluger H, Weiss SA, et al. Collateral damage: insulin-dependent diabetes induced with checkpoint inhibitors. Diabetes 2018;67(8):1471-1480.

12. Ansari MJ, Salama AD, Chitnis T, Smith RN, Yagita H, Akiba H, et al. The programmed death-1 (PD-1) pathway regulates autoimmune diabetes in nonobese diabetic (NOD) mice. J Exp Med 2003;198(1):63-69.

13. Fujisawa R, Haseda F, Tsutsumi C, Hiromine Y, Noso S, Kawabata Y, et al. Low programmed cell death-1 (PD-1) expression in peripheral CD4+T cells in Japanese patients with autoimmune type 1 diabetes. Clin Exp Immunol 2015;180(3):452-457.

14. Hansen E, Sahasrabudhe D, Sievert L. A case report of insulin-dependent diabetes as immune-related toxicity of pembrolizumab: presentation, management and outcome. Cancer Immunol Immunother 2016;65:765-767. 